\title{
Expression profile of circulating microRNAs in patients with ischemic heart failure with moderately reduced left ventricular ejection fraction - pilot study
}

\author{
Damian Kaufmann' ${ }^{1}$, Ludmiła Daniłowicz-Szymanowicz ${ }^{1}$ (D) , \\ Monika Sakowicz-Burkiewicz ${ }^{2}$ (D), Małgorzata Szwoch ${ }^{1}$, \\ Tadeusz Pawełczyk ${ }^{2}$ (D), Grzegorz Raczak ${ }^{1}$
}

${ }^{1}$ Department of Cardiology and Electrotherapy, Medical University of Gdańsk, Poland

2 Department of Molecular Medicine, Medical University of Gdańsk, Poland

\begin{abstract}
Introduction: Heart failure (HF) is a growing global pandemic that affects millions of people around the world. Despite the progress in medicine, diagnosis and treatment of $\mathrm{HF}$ remains problematic. Recently, noncoding micro ribonucleic acids called miRNAs have become significant in the diagnosis and stratification of $\mathrm{HF}$ risk.

Aim: The aim of this study was the attempt to identify the profile of circulating miRNAs specific for ischemic HF with moderately reduced left ventricular ejection fraction (HFmrEF).

Methods and Results: A number of changes in the miRNA profile can characterise patients with ischemic HFmrEF. This is a pilot study before further research on a larger group of patients.

Conclusions: Using the quantitative reverse transcription-polymerase chain reaction (qRT-PCR), serum levels of 84 miRNA were measured and compared between a patient with ischemic HFmrEF and a healthy volunteer. Analysis reveals a down-regulation of let-7f-5p and miR-1-3p, as well as up-regulation of miR-100-5p, miR-10b-5p, miR-125a-5p, miR-140-5p, miR-144-3p, miR-149-5p, miR-15b-5p, miR-183-5p, miR-208b-3p, miR-224-5p, miR-26b-5p, miR-27b-3p, miR-302a-3p, miR-320a, miR-7-5p, miR-99a-5p.

Keywords: heart failure / circulating microRNAs / biomarkers
\end{abstract}

\section{Citation}

Kaufmann D, Daniłowicz-Szymanowicz L, Sakowicz-Burkiewicz M, Szwoch M, Pawełczyk T, Raczak G, et al. Expression profile of circulating microRNAs in patients with ischemic heart failure with moderately reduced left ventricular ejection fraction - pilot study. Eur J Transl Clin Med. 2018;1(2):53-57.

DOI: $10.31373 /$ ejtcm/100543

Corresponding author:

Damian Kaufmann, Department of Cardiology and Electrotherapy, Medical University of Gdańsk, Poland

e-mail: damian.kaufmann@gumed.edu.pl

No external funds.

Available online: www.ejtcm.gumed.edu.pl

Copyright $®$ Medical University of Gdańsk

This is Open Access article distributed under the terms of the Creative Commons Attribution-ShareAlike 4.0 International. 


\section{Introduction}

Heart failure (HF) remains a growing global pandemic that affects at least 37 million people worldwide and counting [1]. Despite significant expenditures on HF treatment, advances in therapy and prevention, mortality remains high and about $50 \%$ of patients with $\mathrm{HF}$ die within 5 years of diagnosis [2], which means the need to continually aim at improving HF diagnosis and treatment. Therefore, new diagnostic and therapeutic methods are being sought for the early identification of high-risk patients and to improve treatment efficacy.

In the last few years, special attention has been paid to microRNA (miRNA). These molecules, usually 21-25 nucleotides in length, are considered to be one of the major post-transcriptional regulators of gene expression. This process is achieved by binding miRNA with the target mRNA in the base pairing mechanism, followed by the induction or repression of translation or mRNA degradation. It is estimated that from $1 \%$ to $5 \%$ of the total genetic material in both humans and animals are genes encoding miRNA [3]. Many reports in the literature confirm that miRNA plays a key role in important processes such as angiogenesis, oncogenesis, fibrosis, apoptosis, cell division or cell differentiation. MiRNA can act intracellularly or can be actively secreted by cells and be involved in cell-cell or cell-tissue communication. Despite high extracellular RNase activity, circulating miRNAs are very stable due to their combination with proteins, lipoproteins, or by packing inside microvesicles [4]. Most miRNAs are located intracellularly, however, they can also be found in various body fluids such as plasma, urine, tears and saliva [5].

Numerous studies have shown that changes in the expression of both intracellular and extracellular miRNAs correlate with various cardiovascular conditions such as myocardial infarction, left ventricular hypertrophy, cardiomyopathy, arrhythmias or HF [6-10]. The unique miRNA expression profile can be a promising marker of various cardiovascular diseases.

\section{MicroRNA biogenesis cellular process}

The miRNA biogenesis cellular process is multistage and includes both nuclear and cytoplasmic processes [11]. The first stage in the cell nucleus is transcription leading to the creation of the original miRNA (pri-miRNA). This process occurs with the participation of RNA polymerase II, which transcribes from the chromosomal DNA pri-miRNA with a length of several thousand base pairs. Thereafter, the microprocessor complex consisting of the DGCR8 nuclear protein and Drosh ri- bonuclease joins the pri-miRNA and processes them into about a 70-nucleotide long double-stranded pinhead structure called a precursor miRNA (pre-miRNA). Subsequently, pre-miRNA by exportin- 5 transferase is actively transported from the nucleus to the cytoplasm where it combines with the Dicer enzyme, which catalyses the reaction leading to the creation of a miRNA-miRNA* duplex with a length of about 21-25 nucleotides. In the last stage, the duplex is separated, and one of the strands (the lead strand) is connected via the Argonaut protein to the RNA-induced silencing complex (RISC). This mature miRNA strand built into RISC has the ability to inhibit gene expression by binding to complementary mRNAs and their subsequent degradation or inhibition of translation [12].

\section{Aim}

The aim of this study was the attempt to identify the profile of circulating miRNAs specific for ischemic HFmrEF.

\section{Materials and methodology}

\section{Comparison of the patient with a healthy volunteer}

Both the patient and the control group were matched in terms of age and sex. The healthy volunteer did not suffer from chronic diseases and did not use any medications. The patient with HFmrEF has a history of 3 percutaneous coronary interventions in the area of the left coronary artery. The last coronary angiography was performed in $\mathbf{2 0 1 0}$ where balloon angioplasty of the left anterior descending coronary artery was performed. There were no haemodynamically significant changes in the remaining coronary arteries. While participating in the study he was in a stable clinical condition, NYHA II with LVEF of $42 \%$. Apart from coronary disease, the patient was burdened with arterial hypertension and hypercholesterolemia. The patient was treated according to the latest guidelines and received a bisoprolol 2,5 $\mathrm{mg}$ q.d, ramipril 2,5 $\mathrm{mg}$ q.d, aspirin $75 \mathrm{mg}$ q.d and rosuvastatin $10 \mathrm{mg}$ q.d.

Using the miScript ${ }^{\mathrm{TM}}$ miRNA Array Human Cardiovascular Disease miRNA, the full profile of 84 miRNA (let-7a-5p, let-7b-5p, let-7c-5p, let-7d-5p, let-7e-5p, let-7f-5p, miR-1-3p, miR-100-5p, miR-103a-3p, miR107, miR-10b-5p, miR-122-5p, miR-124-3p, miR-125a-5p, miR-125b-5p, miR-126-3p, miR-130a-3p, miR-133a-3p, miR-133b, miR-140-5p, miR-142-3p, 
miR-143-3p, miR-144-3p, miR-145-5p, miR-146a-5p, miR-149-5p, miR-150-5p, miR-155-5p, miR-15b-5p, miR-16-5p, miR-17-5p, miR-181a-5p, miR-181b-5p. miR-182-5p, miR-183-5p, miR-185-5p, miR-18b-5p, miR-195-5p, miR-199a-5p, miR-206, miR-208a-3p, miR-208b-3p, miR-21-5p, miR-210-3p, miR-214-3p, miR-22-3p, miR-221-3p, miR-222-3p, miR-223-3p, miR-224-5p, miR-23a-3p, miR-23b-3p, miR-24-3p, miR-253p, miR-26a-5p, miR-26b-5p, miR-27a-3p, miR-27b-3p, miR-29a-3p, miR-29b-3p, miR-29c-3p, miR-302a-3p, miR-302b-3p, miR-30a-5p, miR-30c-5p, miR-30d-5p, miR-30e-5p, miR-31-5p, miR-320a, miR-328-3p, miR-342-3p, miR-365b-3p, miR-378a-3p, miR-423-3p, miR-424-5p, miR-451a, miR-486-5p, miR-494-3p, miR-499a-5p, miR-7-5p, miR-92a-3p, miR-93-5p, miR-98$5 p$, miR-99a-5p) was evaluated from blood serum of the patient and healthy volunteer. For the purpose of the study, written informed consent was obtained from all participants and the study protocol was approved by the local Ethics Committee at the Medical University of Gdańsk.

\section{Molecular methods}

Serum samples. Blood samples were left at room temperature for 30 minutes to allow complete coagulation. Coagulated blood samples were centrifuged at $1,500 \mathrm{~g}$ for 15 minutes at $4{ }^{\circ} \mathrm{C}$ to separate serum. Serum was transferred to a cryotube with care to not disturb the buffy coat and was immediately frozen at $-80^{\circ} \mathrm{C}$ until miRNA extraction.

RNA isolation. Small RNAs was isolated from the serum using the kit the miRNeasy Serum/Plasma kit (Qiagen). Before RNA isolation, all serum samples were thawed completely on ice followed by centrifugation once at $15,000 \mathrm{~g}$ for 15 minutes at $4^{\circ} \mathrm{C}$ to remove remaining cell debris. Then, $200 \mu \mathrm{l}$ of serum sample was carefully transferred to a new $2 \mathrm{ml}$ tube. Serum was mixed with QIAzol lysis reagent in the volumes described within the manufacturer's protocols. Next, 18 fmol of synthetic microRNA - C. elegans miR-39 miRNA was spiked into the all isolation mixtures. Further stages of RNA isolation were carried out in accordance with the manufacturer's protocols. The RNA concentration was measured using a Qubit fluorometer (Invitrogen).

Reverse Transcription. Isolated small RNAs were reverse-transcribed using commercial primers and the miScript II RT kit (Qiagen) according to the manufacturer's protocol. $5 \mu \mathrm{L}$ of RNA ( 30-40 ng) was reverse-transcribed in a $10 \mu \mathrm{L}$ reaction volume for each assay.

MiRNA expression by quantitative real-time PCR. Preamplification was performed using miScript Pre-
AMP PCR Kit and miScript PreAMPPrimer Mix Human Cardiovascular Disease (Qiagen) according to the manufacturer's protocol. Quantitative amplification reaction was performed using the miScript miRNA PCR ArrayHuman Cardiovascular Disease MIHS-113ZF (Qiagen) with miScript SYBR Green PCRkit (Qiagen) in a RightCycler 480 II system (Roche).

\section{Statistical analysis}

Data were normalised by scaling with the mean $\mathrm{Ct}$ of the samples: for each sample the average $\mathrm{Ct}$ of all miRNAs measured in the sample was subtracted from the $\mathrm{Ct}$ of each miRNA. In order to return the signals to a scale which is easier to interpret, a constant (the average Ct over the entire sample set) was added back. Normalised signals were compared between groups in order to find miRNAs which can be used to differentiate between the groups. Fold change was calculated as $2 \Delta$, where $\Delta$ is the absolute difference in median values of the normalised $\mathrm{Ct}$ in the two groups. The $\mathrm{p}$ values are calculated based on a Student's t-test of the replicate $2 \Delta(\Delta \mathrm{Ct})$ values for each gene in the control and treatment group.).

\section{Results}

The conducted analyses showed statistically significant differences in the expression of the following miRNAs: down-regulation of let-7f-5p, miR-1-3p, and up-regulation of miR-100-5p, miR-10b-5p, miR-125a-5p, miR-140-5p, miR-144-3p, miR-149-5p, miR-15b-5p, miR-183-5p, miR-208b-3p, miR-224-5p, miR-26b-5p, miR-27b-3p, miR-302a-3p, miR-320a, miR-7-5p, miR-99a-5p (Table 1). However, there were no differences in the expression of the remaining miRNAs.

\section{Discussion}

The significant development of molecular biology in recent years has led circulating miRNAs to gain an increasing diagnostic and prognostic significance in cardiovascular diseases. Resistance to degradation in the extracellular environment and relatively simple molecular biology methods allowing the detection of miRNA in a quantitative manner in plasma have made them a valuable clinical biomarker. In the available literature, there can be found papers investigating the usefulness of miRNA in diagnosing and stratifying the risk of a wide spectrum of cardiovascular diseases. 
Table 1. MicroRNA (miRNA) with increased level in sera of patients with mid-range ejection fraction

\begin{tabular}{|c|c|c|}
\hline MiRNA & p-value & Fold change \\
\hline let-7f-5p & 0,04505 & $-1,98620$ \\
\hline miR-1-3p & 0,01057 & $-4,95880$ \\
\hline miR-100-5p & 0,00009 & 3,94490 \\
\hline miR-10b-5p & 0,00015 & 3,58010 \\
\hline miR-125a-5p & 0,00000 & 8,16810 \\
\hline miR-140-5p & 0,00000 & 7,94470 \\
\hline miR-144-3p & 0,00050 & 2,90790 \\
\hline miR-149-5p & 0,00001 & 6,36430 \\
\hline miR-15b-5p & 0,00000 & 9,06310 \\
\hline miR-183-5p & 0,00041 & 3,01050 \\
\hline miR-208b-3p & 0,00053 & 2,88790 \\
\hline miR-224-5p & 0,00000 & 15,77970 \\
\hline$m i R-26 b-5 p$ & 0,00001 & 7,21000 \\
\hline miR-27b-3p & 0,00000 & 7,26020 \\
\hline miR-302a-3p & 0,00000 & 14,52030 \\
\hline miR-320a & 0,00000 & 592,22440 \\
\hline miR-7-5p & 0,00149 & 2,44530 \\
\hline miR-99a-5p & 0,00149 & 2,44530 \\
\hline
\end{tabular}

The list includes all miRNAs with an increase of at least 1.2 fold in serum of the mid-range ejection fraction patient vs. serum of the healthy control, sorted by p-value.
Devaux et al proved that miRNA-208b and miR-499 levels are significantly elevated in patients with acute coronary syndrome [7]. A study conducted by Rhodes et al, showed that the decreased level of miR-150 is associated with poor prognosis in patients with pulmonary arterial hypertension [13]. Whereas, in the study of Liu et al, the reduction in miR-150 level was statistically significant among the group of patients with atrial fibrillation, in comparison with the healthy control group [9]. Chen et al, comparing the miRNA expression profile of a healthy control group with patients with HF (both with preserved systolic function (HFPEF) and with impaired left ventricular systolic function) showed that plasma levels of three miRNAs (miR-3135b, miR-3908, and miR-5571-5p) can be used as biomarkers [6].

In our study, the identified profile of 84 circulating miRNAs could be characteristic for patients with ischemic HFmrEF. From among the 84 examined circulating miRNAs, 18 of them showed significant differences in expression compared to the healthy control (Table 1). According to the author's knowledge, this is the first study that characterizes the miRNA profile for such patients. Wong et al, who compared the miRNA expression profile in $\mathrm{HF}$ patients with preserved and reduced left ventricular ejection fraction in relation to healthy subjects, showed miR-1233, miR-183-3p, miR-190a, miR-193b levels $-3 p$, miR-193b-5p, miR-211-5p, miR494, and miR-671-5p were significantly different between patients with $\mathrm{HF}$ and the control group, while miR-125a-5p, miR-183-3p levels, miR-193b-3p, miR211-5p, miR-494, miR-638 and miR-671-5p differentiated the group of patients with reduced left ventricular ejection fraction and the control group [14]. Comparing Wong's results and ours one can notice that there is compliance in the up-regulation of only two miRNA's: miR-183 and miR-125a-5p. There may be several reasons for the low compliance of these two studies. In above mentioned paper the expression profile of more miRNAs (806 miRNA vs. 84 miRNA) was determined, as well as the aetiology of HF was not homogeneous. In Wong's et al the expression profile of more miRNAs (806 miRNA vs. 84 miRNA) was determined, as well as the aetiology of HF was not homogeneous. Another study conducted by Goren et al, comparing serum miRNA concentrations between patients with $\mathrm{HF}$ and left ventricular ejection fraction 30\% (23-33\%) and a healthy control group showed a significant increase in miR-423-5p, miR-320a, miR-22 and miR-92b in people with HF [15]. Likewise, our study also showed an increase in miR-320a expression in serum. Compliance has not been shown in the expression of other miRNA, which most likely was due to the selection of the stu- 
died group as well as the fact that only some of them had an ischemic HF aetiology. Additionally, in our study we did not assess the concentrations of miR-92b and mi-423-5b, which in the referenced study showed statistically significant differences.

Much has been published about miRNA in HF. However, the obtained results are heterogeneous, and more HF-related miRNAs are being constantly identified. This is most likely the result of complex pathophysiological processes leading ultimately to the clinical manifestation of the disease. Therefore, it seems important to conduct further research, including careful selection of the studied population, as well as standardisation of analytical processes in order to obtain more reproducible results.

\section{Limitations of the study}

There are several limitations of our study. First of all, it has been conducted on a small number of patients and further research is needed in this area. Second, although the 84 miRNA panel was used for analysis, it should be emphasized that there are considerably more of these molecules and many have not been included in the study.

\section{Conclusions}

Our study identified the profile of circulating miRNAs that distinguished a patient with HFmrEF from a healthy person. In patients with HFmrEF there is a down-regulation in let-7f-5p and miR-1-3p, as well as an up-regulation in expression of miR-100-5p, miR-10b-5p, miR-125a-5p, miR-140-5p, miR-144-3p, miR-149-5p, miR-15b-5p, miR-183-5p, miR-208b -3p, miR-224-5p, miR-26b-5p, miR-27b-3p, miR-302a-3p, miR-320a, miR-7-5p, miR-99a-5p. Further studies of circulating miRNAs in patients with HFmrEF may offer additional diagnostic and prognostic applications that may be used in clinical practice. This is the first pilot study conducted on a small group of participants, which requires research on a larger group of people in order to confirm the results.

References

1. Bui AL, Horwich TB, Fonarow GC. Epidemiology and risk profile of heart failure. Nat Rev Cardiol. 2011;8(1):30-41.

2. Mozaffarian D, Benjamin EJ, Go AS, Arnett DK, Blaha MJ, Cushman M, et al. Heart Disease and Stroke Statistics - 2015 Update. Circulation. 2015 Jan 27;131(4):e38-360.

3. Berezikov E, Guryev V, van de Belt J, Wienholds E, Plasterk RHA, Cuppen E. Phylogenetic shadowing and computational identification of human microRNA genes. Cell. 2005;120(1):21-4.

4. Rachagani S, Macha MA, Menning MS, Dey P, Pai P, Smith LM, et al. Changes in microRNA (miRNA) expression during pancreatic cancer development and progression in a genetically engineered KrasG12D; Pdx1-Cre mouse (KC) model. Oncotarget. 2015;6(37):40295.

5. Weber JA, Baxter DH, Zhang S, Huang DY, Huang KH, Lee MJ, et al. The microRNA spectrum in 12 body fluids. Clin Chem. 2010;56(11):1733-41.

6. Chen F, Yang J, Li Y, Wang H. Circulating microRNAs as novel biomarkers for heart failure. Hell J Cardiol. 2018 Jul;59(4):209-14.

7. Devaux Y, Vausort M, McCann GP, Kelly D, Collignon O, Ng LL, et al. A panel of 4 microRNAs facilitates the prediction of left ventricular contractility after acute myocardial infarction. PLoS One. 2013;8(8):e70644.

8. Gupta SK, Bang C, Thum T. Circulating microRNAs as biomarkers and potential paracrine mediators of cardiovascular disease. Circ Genomic Precis Med. 2010;3(5):484-8.

9. Liu Z, Zhou C, Liu Y, Wang S, Ye P, Miao X, et al. The expression levels of plasma micoRNAs in atrial fibrillation patients. PLoS One. 2012;7(9):e44906.

10. Van Rooij E, Sutherland LB, Liu N, Williams AH, McAnally J, Gerard RD, et al. A signature pattern of stress-responsive microRNAs that can evoke cardiac hypertrophy and heart failure. Proc Natl Acad Sci. 2006;103(48):1825-60.

11. Lee $\mathrm{Y}$, Jeon K, Lee J, Kim S, Kim VN. MicroRNA maturation: stepwise processing and subcellular localization. EMBO J. 2002;21(17):4663-70.

12. Rana TM. Illuminating the silence: understanding the structure and function of small RNAs. Nat Rev Mol cell Biol. 2007;8(1):23.

13. Rhodes CJ, Wharton J, Boon RA, Roexe T, Tsang H, Wojciak-Stothard B, et al. Reduced microRNA-150 is associated with poor survival in pulmonary arterial hypertension. Am J Respir Crit Care Med. 2013;187(3):294-302.

14. Wong LL, Armugam A, Sepramaniam S, Karolina DS, Lim KY, Lim JY, et al. Circulating microRNAs in heart failure with reduced and preserved left ventricular ejection fraction. Eur J Heart Fail. 2015;17(4):393-404.

15. Goren Y, Kushnir M, Zafrir B, Tabak S, Lewis BS, Amir O. Serum levels of microRNAs in patients with heart failure. Eur J Heart Fail. 2012;14(2):147-54. 